\title{
SYNTHESIS AND EVALUATION OF SOME BENZOTHIAZOLE DERIVATIVES AS ANTIDIABETIC AGENTS
}

\author{
SUNIL KUMAR ${ }^{\text {* }}$, D. S. RATHORE ${ }^{a}$, GOPAL GARG ${ }^{a}$, KAPIL KHATRI ${ }^{b}$, RAHUL SAXENA ${ }^{b}$, SANJEEV K. SAHU \\ aNIMS Institute of Pharmacy, NIMS University, Jaipur (Rajasthan) India, bRavishankar College of Pharmacy, Bhopal (M. P.) India \\ Email: yagnikvns@gmail.com
}

Received: 28 Jul 2016 Revised and Accepted: 07 Dec 2016

\section{ABSTRACT}

Objective: The objective of the present research investigation involves synthesis and biological evaluation of antidiabetic activity of benzothiazole derivatives.

Methods: A novel series of benzothiazole derivatives 7(a-l) were synthesised and synthesised compounds were characterised for different physical and chemical properties like molecular formula, molecular weight, melting point, percentage yield, Rf value, IR, ${ }^{1} \mathrm{HNMR},{ }^{13} \mathrm{CNMR}$ and mass spectroscopy. The newly synthesised benzothiazole derivatives were subsequently assayed in vivo to investigate their hypoglycemic activity by the alloxan-induced diabetic model in rats.

Results: All the synthesised derivatives showed significant biological efficacy. The compound $7 \mathrm{~d}$ at $350 \mathrm{mg} / \mathrm{kg}$ exerted maximum glucose lowering effects whereas $7 c$ showed minimum glucose lowering effects. All the compounds were effective, and experimental results were statistically significant at $\mathrm{p}<0.01$ and $\mathrm{p}<0.05$ level

Conclusion: From the results, it is clear that compound $7 \mathrm{~d}$ demonstrated potent anti-diabetic activity and would be of better use in drug development to combat the metabolic disorder in future.

Keywords: Diabetes, Substituted benzthiazole derivatives, Thiazolidinedione, Hypoglycemic activity

(C) 2017 The Authors. Published by Innovare Academic Sciences Pvt Ltd. This is an open access article under the CC BY license (http://creativecommons.org/licenses/by/4. 0/] DOI: http://dx.doi.org/10.22159/ijpps.2017v9i2.14359

\section{INTRODUCTION}

Diabetes mellitus is an endocrinological and metabolic disorder with an increasing global prevalence and incidence. High blood glucose levels are symptomatic of diabetes mellitus as a consequence of inadequate pancreatic insulin secretion or poor insulin-directed mobilisation of glucose by target cells [1]. The world health organisation (WHO) estimates that more than 220 million people worldwide have diabetes and this number is likely to double by 2030 (WHO, 2009). Several drugs such as sulfonylurea and biguanides are presently available to reduce hyperglycemia in diabetes mellitus.

These drugs have a number of side effects and thus searching for a new class of compounds is crucial to overcoming these problems. Heterocyclic compounds are the mainstay of antidiabetic therapy for many years [2]. Also, certain hypoglycemic plants may also be usefu to develop evidence-based alternative medicine to cure different kinds of diabetes in man and animals [3]

Benzothiazole is a weak heterocyclic base, having varied biological activities and of great scientific interest nowadays. Benzothiazoles are fused membered rings, which contain the heterocycles bearing thiazole. Sulphur and nitrogen atoms constitute the core structure of thiazole and many pharmacologically and biologically active compounds [4]. Benzothiazole ring system is present in various marine and terrestrial natural compounds, which have useful biological activities [5].

The benzthiazole derivatives have demonstrated efficiency in biological fields such as antitumor, antitubercular, antimalarial, anticonvulsant, anthelmintic, analgesic, anti-inflammatory, antifungal, a topical carbonic anhydrase inhibitor and an antihypoxic. They are broadly found in bioorganic and medicinal chemistry with applications in drug discovery and development for the treatment of diabetes [6, 7]. Due to it's potent and significant biological activities, it has great pharmaceutical importance $[8,9]$
Benzothiazole derivatives containing benzimidazole and imidazoline ring have diverse chemical reactivity along with a broad spectrum of biological activity [10]. In view of this literature, it was of significant interest to synthesise the benzothiazole derivatives with an aim to obtain potent biologically active and safe anti-diabetic agents.

\section{MATERIALS AND METHODS}

All the chemicals were of synthetic grade and commercially procured from hi media chemicals, Mumbai (Maharashtra) INDIA. ERBA diagnostic kit was used for the determination of glucose. Melting points were determined on a tempo capillary melting point apparatus in open capillary tube. UV-visible spectrophotometric determination was carried on systronics 2203. All FTIR spectra were recorded (vmax in $\mathrm{cm}^{-1}$ ) on bruker tensor 27 FT-IR spectrometer. ${ }^{1} \mathrm{H}-\mathrm{NMR}$ (proton nuclear magnetic resonance) spectrum were recorded at $300 \mathrm{MHz}$, after dissolving in a suitable solvent (DMSO, $\mathrm{CDCl}_{3}$ or $\mathrm{D}_{2} \mathrm{O}$ ) on bruker avance II $400 \mathrm{MHz}$, USA FT-NMR spectrometer using tetramethylsilane as internal standard and chemical shifts $(\delta)$ are reported in parts per million (ppm). ${ }^{13} \mathrm{C}$ NMR was also recorded on bruker avance II FT-NMR spectrometer at a frequency of $100 \mathrm{MHz}$. The spin multiplicities are indicated by symbols, s (singlet), d (doublet), t (triplet), q (quartet), m (multiple) and br (broad). Mass spectra were recorded on Waters UPLC-TQD mass spectrometer using electrospray ionisation (ESI) technique.

The purity of the compounds was ascertained by thin layer chromatography (TLC) and elemental analysis. Plates for TLC were prepared with silica gel $\mathrm{G}$ and activated at $110{ }^{\circ} \mathrm{C}$ for $30 \mathrm{~min}$. Iodine vapours were used to develop the TLC plates. Elemental analyses were performed on a vario EL-III analyser.

General method of synthesis for benzthiazole derivatives

All the compounds were synthesised by using a synthetic route given in scheme as follows: 
Table 1: Benzothiazole derivatives with different substitutions

\begin{tabular}{|c|c|c|c|c|}
\hline S. No. & Compound code & $\mathbf{R}_{1}$ & $\mathbf{R}_{2}$ & $\mathbf{R}_{3}$ \\
\hline 1. & $7 a$ & $\mathrm{CH}_{3}$ & $\mathrm{H}$ & $\mathrm{C}_{6} \mathrm{H}_{5-}$ \\
\hline 2. & $7 \mathrm{~b}$ & $\mathrm{CH}_{3}$ & $\mathrm{H}$ & $\mathrm{pOHC}_{6} \mathrm{H}_{4-}$ \\
\hline 3. & $7 \mathrm{c}$ & $\mathrm{CH}_{3}$ & $\mathrm{H}$ & $\mathrm{pOCH}_{3} \mathrm{C}_{6} \mathrm{H}_{4-}$ \\
\hline 4. & $7 d$ & $\mathrm{CH}_{3}$ & $\mathrm{H}$ & $\mathrm{m} \mathrm{NO}_{2} \mathrm{C}_{6} \mathrm{H}_{4-}$ \\
\hline 5. & $7 e$ & $\mathrm{NO}_{2}$ & $\mathrm{H}$ & $\mathrm{C}_{6} \mathrm{H}_{5-}$ \\
\hline 6. & $7 f$ & $\mathrm{NO}_{2}$ & $\mathrm{H}$ & $\mathrm{pOHC}_{6} \mathrm{H}_{4-}$ \\
\hline 7. & $7 \mathrm{~g}$ & $\mathrm{NO}_{2}$ & $\mathrm{H}$ & $\mathrm{pOCH}_{3} \mathrm{C}_{6} \mathrm{H}_{4-}$ \\
\hline 8. & $7 \mathrm{~h}$ & $\mathrm{NO}_{2}$ & $\mathrm{H}$ & $\mathrm{m} \mathrm{NO}_{2} \mathrm{C}_{6} \mathrm{H}_{4-}$ \\
\hline 9. & $7 i$ & $\mathrm{H}$ & $\mathrm{NO}_{2}$ & $\mathrm{C}_{6} \mathrm{H}_{5-}$ \\
\hline 10. & $7 \mathrm{j}$ & $\mathrm{H}$ & $\mathrm{NO}_{2}$ & $\mathrm{pOHC}_{6} \mathrm{H}_{4-}$ \\
\hline 11. & $7 \mathrm{k}$ & $\mathrm{H}$ & $\mathrm{NO}_{2}$ & $\mathrm{pOCH}_{3} \mathrm{C}_{6} \mathrm{H}_{4-}$ \\
\hline 12. & 71 & $\mathrm{H}$ & $\mathrm{NO}_{2}$ & $\mathrm{~m} \mathrm{NO}_{2} \mathrm{C}_{6} \mathrm{H}_{4-}$ \\
\hline
\end{tabular}
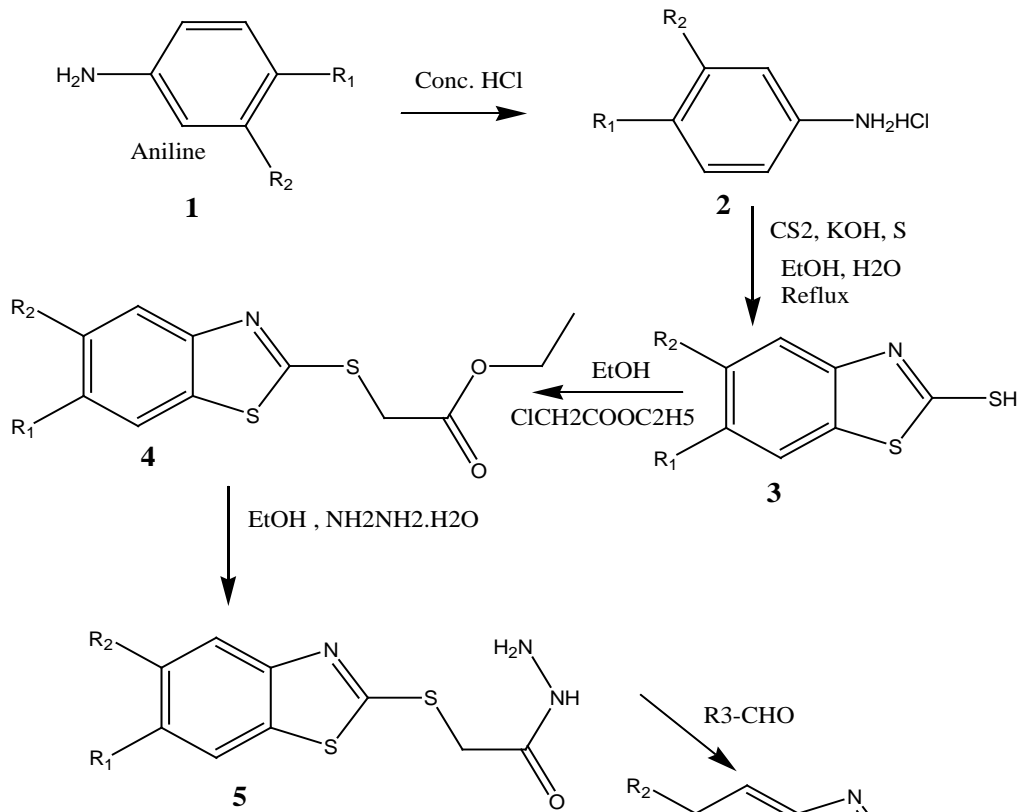

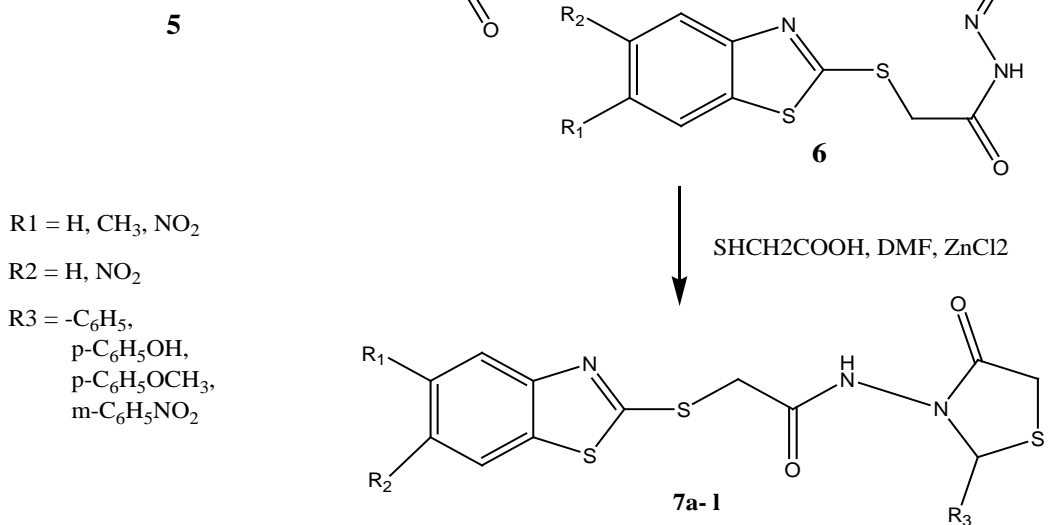

Fig. 1: Scheme of synthesis for benzothiazole derivatives

\section{Synthesis of substituted aniline hydrochloride salt [Compound 2]}

Aniline $(0.1 \mathrm{~mol})$ was taken in a round bottom flask and to it a mixture of $\mathrm{HCl}(9 \mathrm{ml})$ and water $(25 \mathrm{ml})$ was added. Then the solution was heated for about $30 \mathrm{~min}$ and then cooled at room temp. Further, ammonium thiocyanate $(0.1 \mathrm{~mol})$ was added to the reaction mixture, refluxed for $4 \mathrm{~h}$ and was cooled. The precipitate thus obtained was filtered, washed with water, dried and crystallised from ethanol.

\section{Synthesis of substituted benzo[d]thiazole-2-thiol [Compound 3]}

A mixture of compound $\mathbf{1}(0.0025 \mathrm{~mol})$, potassium hydroxide $(0.75$ $\mathrm{gm})$ in water $(6 \mathrm{ml})$ and carbon disulphide $(1.6 \mathrm{~g}, 0.01 \mathrm{~mol})$ in presence of sulphur ( $5 \mathrm{ml})$ and absolute ethanol $(30 \mathrm{ml})$ was heated under reflux for $2 \mathrm{~h}$ at $280-285{ }^{\circ} \mathrm{C}$ and $600-700 \mathrm{psi}$ pressure. The reaction mixture was cooled, filtered and the filtrate was acidified with a dilute hydrochloric acid, the product thus obtained was collected and recrystallized from ethanol [11]

Synthesis of substituted ethyl 2-(benzo[d]thiazol-2-ylthio) acetate [Compound 4]

2-mercaptobenzothiazole $(0.2 \mathrm{~mol})$ and ethyl chloroacetate $(0.02$ $\mathrm{mol})$ in dry acetone in the presence of $\mathrm{K}_{2} \mathrm{CO}_{3}(20 \mathrm{~g})$ was refluxed for $10 \mathrm{~h}$ and the reaction mixture was poured into ice and neutralised with dil. $\mathrm{HCl}$, the solid thus obtained was washed several times with water and recrystallized from chloroform. 
Synthesis of substituted 2-(benzo[d]thiazol-2-ylthio) acetohydrazide [Compound 5]

Into a clean, dry $100 \mathrm{ml}$ round bottomed flask, the ethyl-2benzothiazole carboxylate $(0.01 \mathrm{~mol})$ was dissolved in ethanol $(60$ $\mathrm{ml})$. The hydrazine hydrate $(0.02 \mathrm{~mol})(99 \%)$ was added drop by drop with constant stirring and the content were refluxed for $8 \mathrm{~h}$ and thus cooled to room temperature.

Synthesis of substituted 2-(benzo[d]thiazol-2-ylthio)-N'-methyleneacetohydrazide [Compound 6]

A mixture of compound 5 (1 g, $0.0034 \mathrm{~mol})$ and different benzaldehydes $(0.0034 \mathrm{~mol})$ were taken with absolute ethanol (20 $\mathrm{ml}$ ) and was refluxed for $3 \mathrm{~h}$. The solvent was evaporated and the residue was recrystallized from ethanol [12].

\section{Synthesis of benzthiazole derivatives [Compound 7a-1]}

A mixture of the previous compound $(0.01 \mathrm{~mol})$ and mercapto acetic acid $(0.012 \mathrm{~mol})$ in DMF $(25 \mathrm{ml})$ containing a pinch of anhydrous $\mathrm{ZnCl}_{2}$ was refluxed for $8 \mathrm{~h}$. The reaction mixture was then cooled and poured into ice-cold water. The resulting solid was filtered, washed several times with water and then crystallised from DMF [13].

\section{Determination of physical properties of synthesised derivatives}

The physical properties were determined for all the synthesised derivatives that include molecular formula, molecular weight, melting point, percentage yield and $\mathrm{Rf}$ value.

\section{Pharmacological evaluation of synthesised derivatives}

\section{Determination of acute toxicity (OECD 425, 2008) [14]}

The acute toxicity of synthesised benzothiazole derivatives was determined by using female albino rats (200-250 g) which were maintained under the standard conditions. The acclimatised animals $(n=6)$ were kept fasting with water ad libitum for $12 \mathrm{~h}$ prior to the experiment. The animals were administered with single dose of test compound at a dose of $2000 \mathrm{mg} / \mathrm{kg}$ and observed for their mortality during $14 \mathrm{~d}$ period for toxicity study. The doses were increased up to $1000 \mathrm{mg} / \mathrm{kg}$ and rats were observed up to $02 \mathrm{w}$ for their behavioural, economical and neurological profiles except for slight depression in their activity. No such signs, symptoms and mortality were observed even after $14 \mathrm{~d}$. Hence the $\mathrm{LD}_{50}$ cut off the value of the test compounds was fixed at $350 \mathrm{mg} / \mathrm{kg}$ and the same dose was considered for evaluation of the antidiabetic activity. All the animal experiments were conducted by the approval of Institutional Animal Ethics Committee (Approval No. SBRL/IAEC/July2015/12), Sapience Bioanalytical Laboratory Bhopal, Madhya Pradesh, India

Assessments of anti-diabetic activity in alloxan-induced diabetic rats [15]

\section{Induction of experimental diabetes by alloxan monohydrate}

The fresh solution of alloxan monohydrate in normal saline was administered p. o. into fasted rats at a dose of $120 \mathrm{mg} / \mathrm{kg}$ body weight $[16,17]$. After alloxan administration (i. p.), rats were given
$5 \%(\mathrm{w} / \mathrm{v})$ dextrose solution in feeding bottles for next $24 \mathrm{~h}$ in their cages to to prevent hypoglycaemia. The animals showing blood glucose range of 200-400 mg dL-1 were used for the experiment and the hyperglycemia was confirmed after $72 \mathrm{~h}$ of alloxan monohydrate administration (i. p.). The animals were also observed for consistent hyperglycaemia (fasting blood glucose) between $200-400 \mathrm{mg} / \mathrm{dl}$ up to $14 \mathrm{~d}$.

\section{Experimental design}

Animals were divided into fourteen groups of 6 animals in each $(n=6)$. Group 1 diabetic animals received $1 \mathrm{ml}$ of $0.5 \%$ carboxymethyl cellulose as a control group; Group 2 diabetic animals received Glibenclamide $20 \mathrm{mg} / \mathrm{kg}$ as a standard group; Groups (3-14) diabetic animals received compounds 7a-7l in a single dose of $350 \mathrm{mg} / \mathrm{kg}$ body weight p. o. respectively for $14 \mathrm{~d}$ continuously.

\section{Blood glucose measurement}

Blood glucose level was monitored by a tail dipping method. The blood sample was dropped on the dextrostrix reagent pad. The strip was inserted into microprocessor digital blood glucometer, and reading was noted. The blood glucose level was monitored at $0 \mathrm{~h}, 3 \mathrm{~h}$, $7 \mathrm{~h}, 10 \mathrm{~h}$ respectively.

\section{Statistical analysis}

The values were expressed as mean \pm SEM Data were analysed using One-way ANOVA followed by Tukey-Kramer test. The values were considered to be significant at $\mathrm{p}<0.05$ and $\mathrm{p}<0.01$ level.

\section{RESULTS AND DISCUSSION}

All the benzthiazole derivatives $7(\mathrm{a}-\mathrm{l})$ were synthesised by the given scheme and reaction process was monitored by thin layer chromatography method using silica gel-G stationary phase, ethyl acetate: ethanol (2:3) as mobile phase, and detecting the spots with iodine vapours. All the constant physical data were characterised for all the synthesised derivatives that are given in the synthesised derivatives were also confirmed by FTIR, ${ }^{1} \mathrm{H}$ NMR, $\mathrm{C}^{13} \mathrm{NMR}$ and mass spectroscopy method. The FTIR spectrums demonstrated the significant peaks at $3270-3260 \mathrm{~cm}^{-1}(\mathrm{~N}-\mathrm{H}$ strech.), $1720-1710 \mathrm{~cm}$ ${ }^{1}$ (cyclic C=0 strech.), $1660 \mathrm{~cm}^{-1}$ (amide $\mathrm{C}=0$ stretch.), $1320-1310 \mathrm{~cm}-$ ${ }_{1}^{1}$ (C-N Strech), 695-685 $\mathrm{cm}^{-1}$ (C-S stretch) $\mathrm{cm}^{-1}$.

The different substitutions on phenyl ring were confirmed through FTIR spectrum peaks at $3480-3460 \mathrm{~cm}^{-1}(\mathrm{O}-\mathrm{H}$ stretch.) for hydroxyl group, 1250 (asym. C-0 strech.) and 1038 (sym. C-0 stretch.) for methoxy group, 1385-90 (sym. $\mathrm{CH}_{3}$ bending) and $1330-40$ (sym $\mathrm{NO}_{2}$ bending) for nitro group substitution. The proton NMR spectrums also confirmed the different substitutions on phenyl as well as benzothiazole ring distinct due to change in the environment of protons. Similarly, the environment surrounding carbon atoms were also changed which were confirmed through the $\mathrm{C}^{13} \mathrm{NMR}$. The mass spectroscopy studies confirmed the molecular weight of derivatives through molecular ion peak on the mass spectrum (i.e. peak at highest $\mathrm{m} / \mathrm{e}$ ).

Table 2: Physical constant data of synthesised derivatives

\begin{tabular}{|c|c|c|c|c|c|}
\hline $\begin{array}{l}\text { Properties } \rightarrow \\
\text { Code of derivatives } \downarrow\end{array}$ & Molecular formula & Molecular weight & Melting point in ${ }^{\circ} \mathrm{C}$ & \% Yield & Retention factor \\
\hline $7 a$ & $\mathrm{C}_{19} \mathrm{H}_{17} \mathrm{~N}_{3} \mathrm{O}_{2} \mathrm{~S}_{3}$ & 415.55 & $168-170$ & 62.5 & 0.58 \\
\hline $7 b$ & $\mathrm{C}_{19} \mathrm{H}_{17} \mathrm{~N}_{3} \mathrm{O}_{3} \mathrm{~S}_{3}$ & 431.55 & $188-189$ & 68.7 & 0.62 \\
\hline $7 \mathrm{c}$ & $\mathrm{C}_{20} \mathrm{H}_{19} \mathrm{~N}_{3} \mathrm{O}_{3} \mathrm{~S}_{3}$ & 445.58 & $210-212$ & 72.3 & 0.64 \\
\hline $7 d$ & $\mathrm{C}_{19} \mathrm{H}_{16} \mathrm{~N}_{4} \mathrm{O}_{4} \mathrm{~S}_{3}$ & 460.55 & $276-278$ & 76.2 & 0.72 \\
\hline $7 e$ & $\mathrm{C}_{18} \mathrm{H}_{14} \mathrm{~N}_{4} \mathrm{O}_{4} \mathrm{~S}_{3}$ & 446.52 & $165-167$ & 59.5 & 0.63 \\
\hline $7 f$ & $\mathrm{C}_{18} \mathrm{H}_{14} \mathrm{~N}_{4} \mathrm{O}_{5} \mathrm{~S}_{3}$ & 462.52 & 184-186 & 63.2 & 0.68 \\
\hline $7 \mathrm{~g}$ & $\mathrm{C}_{19} \mathrm{H}_{16} \mathrm{~N}_{4} \mathrm{O}_{5} \mathrm{~S}_{3}$ & 476.55 & 207-209 & 69.1 & 0.71 \\
\hline $7 \mathrm{~h}$ & $\mathrm{C}_{18} \mathrm{H}_{13} \mathrm{~N}_{5} \mathrm{O}_{6} \mathrm{~S}_{3}$ & 491.52 & $273-275$ & 74.8 & 0.78 \\
\hline $7 \mathrm{i}$ & $\mathrm{C}_{18} \mathrm{H}_{14} \mathrm{~N}_{4} \mathrm{O}_{4} \mathrm{~S}_{3}$ & 446.52 & $161-163$ & 56.8 & 0.61 \\
\hline $7 \mathrm{j}$ & $\mathrm{C}_{18} \mathrm{H}_{14} \mathrm{~N}_{4} \mathrm{O}_{5} \mathrm{~S}_{3}$ & 462,52 & 179-181 & 61.3 & 0.69 \\
\hline $7 \mathrm{k}$ & $\mathrm{C}_{19} \mathrm{H}_{16} \mathrm{~N}_{4} \mathrm{O}_{5} \mathrm{~S}_{3}$ & 476.55 & $202-204$ & 67.7 & 0.70 \\
\hline 71 & $\mathrm{C}_{18} \mathrm{H}_{13} \mathrm{~N}_{5} \mathrm{O}_{6} \mathrm{~S}_{3}$ & 491.52 & $269-271$ & 71.9 & 0.77 \\
\hline
\end{tabular}


(A) 2-(5-methylbenzo[d]thiazol-2-ylthio)-N-(4-oxo-2-phenylthiazolidin-3-yl)acetamide [compound 7a]

Yield: $62.5 \%$; M. P. $168-170{ }^{\circ} \mathrm{C}$; Anal. Cal. for $\mathrm{C}_{19} \mathrm{H}_{17} \mathrm{~N}_{3} \mathrm{O}_{2} \mathrm{~S}_{3}$ : C, 54.92; H, 4.12; N, 10.11; 0, 7.70; S, 23.15\%; found: C, 54.92; H, 4.14; $\mathrm{N}, 10.06 ; 0,7.72 ; \mathrm{S}, 23.13 \%$;

\section{FT-IR spectroscopy}

FT-IR (vmax): 3265 (N-H strech.), 3030 (aromatic C-H strech.), 2960 (asym. aliphatic C-H strech.), 2858 (sym. aliphatic C-H strech.), $1670-2000$ (overtone for substitution on aromatic ring), 1710 (cyclic C=0 strech.), 1660 (amide C=0 stretch.), 1598 (phenyl ring stretch.), 1578 ( $\mathrm{N}-\mathrm{H}$ bending), 1510 (phenyl C-H out of plane bending), $1465\left(\mathrm{CH}_{2}\right.$ bending), 1456 (asym $\mathrm{CH}_{3}$ bending), 1389 (sym. $\mathrm{CH}_{3}$ bending), 1315 (C-N Strech), 690 (C-S stretch) $\mathrm{cm}^{-1}$.

\section{H-NMR spectroscopy}

${ }^{1} \mathrm{H}-\mathrm{NMR}\left(\mathrm{CDCl}_{3}\right)(\delta, \mathrm{ppm}): 7.94(\mathrm{~s}, 1 \mathrm{H},-\mathrm{CONH}-), 7.89-7.78(\mathrm{~m}, 3 \mathrm{H}$, benzthiazole ring protons), 7.36-7.26 (m, $5 \mathrm{H}$, phenyl ring protons), $5.92(\mathrm{~s}, 1 \mathrm{H}$, thiazole ring protons at phenyl linkage), 4.35 (s, $2 \mathrm{H}$,$\left.\mathrm{SCH}_{2}-\right), 3.95-3.82\left(\mathrm{~s}, 2 \mathrm{H}\right.$, thiazole ring protons), $2.33\left(\mathrm{~s}, 3 \mathrm{H}, \mathrm{CH}_{3}\right.$ at benzthiazole linkage).

\section{${ }^{13} \mathrm{C}$-NMR spectroscopy}

${ }^{13} \mathrm{C}$-NMR $\left(\mathrm{CDCl}_{3}\right)(\delta, \mathrm{ppm}): 170.3(\mathrm{C}=0$, amide carbon), $168.6(\mathrm{C}=0$ thiazole ring carbon), $164.2(\mathrm{C}=\mathrm{N}$ bezthiazole ring carbon at sulfur linkage), 150.5 (C-N benzthiazole ring carbon), 139.2 (phenyl ring carbon at thiazole linkage), 134.2-135.0 (bezthiazole ring carbons at sulfur and methyl linkage), 127.2-128.8 (phenyl ring carbons), 121.5-126.6 (benzthiazole ring carbons at proton linkage), 57.3 (thiazole ring carbon at phenyl linkage), 40.9 (-S-CH $2-\mathrm{CONH}-), 35.7$ (thiazole ring carbon at- $\mathrm{C}=0$ linkage), 23.9 (methyl carbon at benzthiazole ring)

\section{Mass spectroscopy}

Molecular ion peak demonstrated on mass spectrum and reported m/e (ESI): $415\left(\mathrm{M}^{+}\right)$.

(B) N-(2-(4-hydroxyphenyl)-4-oxothiazolidin-3-yl)-2-(5methylbenzo[d]thiazol-2-ylthio) acetamide [compound 7b]

Yield: 68.7 \%; M. P. 188-189 ${ }^{\circ} \mathrm{C}$; Anal. Cal. for $\mathrm{C}_{19} \mathrm{H}_{17} \mathrm{~N}_{3} \mathrm{O}_{3} \mathrm{~S}_{3}$ : C, 52.88; H, 3.97; N, 9.74; O, 11.12; S, 22.29\%; found: C, 52.91; H, 3.95; N, 9.76; 0, 11.14; S, 22.24\%;

\section{FT-IR spectroscopy}

FT-IR (vmax): 3465 (O-H stretch.), 3268 (N-H strech.), 3033 (aromatic C-H strech.), 2962(asym. aliphatic C-H strech.), 2861 (sym. aliphatic C-H strech.), 1670-2000 (overtone for substitution on aromatic ring), 1714 (cyclic $\mathrm{C}=0$ strech.), 1662 (amide $\mathrm{C}=0$ stretch.), 1603 (phenyl ring stretch.), 1580 (N-H bending), 1512 (phenyl C-H out of plane bending), $1466\left(\mathrm{CH}_{2}\right.$ bending), 1458 (asym $\mathrm{CH}_{3}$ bending), 1388 (sym. $\mathrm{CH}_{3}$ bending), 1317 (C-N Strech), 692 (C-S stretch) $\mathrm{cm}^{-1}$.

\section{${ }^{1} \mathrm{H}$-NMR spectroscopy}

${ }^{1} \mathrm{H}-\mathrm{NMR}\left(\mathrm{CDCl}_{3}\right)(\delta, \mathrm{ppm}): 7.94(\mathrm{~s}, 1 \mathrm{H},-\mathrm{CONH}-), 7.89-7.76(\mathrm{~m}, 3 \mathrm{H}$, benzthiazole ring protons), 7.35-6.63 $(\mathrm{m}, 4 \mathrm{H}$, phenyl ring protons), $5.91(\mathrm{~s}, 1 \mathrm{H}$, thiazole ring protons at phenyl linkage), $5.35(\mathrm{~s}, 1 \mathrm{H}$,$\left.\mathrm{C}_{6} \mathrm{H}_{4}-\mathrm{OH}\right), 4.35\left(\mathrm{~s}, 2 \mathrm{H},-\mathrm{SCH}_{2}-\right), 3.95-3.83(\mathrm{~s}, 2 \mathrm{H}$, thiazole ring protons), 2.34 (s, $3 \mathrm{H}, \mathrm{CH}_{3}$ at benzthiazole linkage).

\section{${ }^{13} \mathrm{C}$-NMR spectroscopy}

${ }^{13} \mathrm{C}$-NMR $\left(\mathrm{CDCl}_{3}\right)(\delta, \mathrm{ppm}): 170.4(\mathrm{C}=\mathrm{O}$, amide carbon $), 168.8(\mathrm{C}=0$ thiazole ring carbon), $164.4(\mathrm{C}=\mathrm{N}$ bezthiazole ring carbon at sulfur linkage), 151.3 (C-N benzthiazole ring carbon), 156.9 (phenyl ring carbon at hydroxyl linkage), 134.3-135.8 (bezthiazole ring carbons at sulfur and methyl linkage), 131.8 (phenyl ring carbon at thiazole linkage), 121.5-126.6 (benzthiazole ring carbons at proton linkage), 115.8-125.2 (phenyl ring carbons), 57.8 (thiazole ring carbon at phenyl linkage), 41.2 (-S- $\mathrm{CH}_{2}$-CONH-), 36.4 (thiazole ring carbon at$\mathrm{C}=0$ linkage), 24.5 (methyl carbon at benzthiazole ring).

\section{Mass spectroscopy}

Molecular ion peak demonstrated on mass spectrum and reported m/e (ESI): $432\left(\mathrm{M}^{+}\right)$.

(C) N-(2-(4-methoxyphenyl)-4-oxothiazolidin-3-yl)-2-(5methylbenzo[d] thiazol-2-ylthio) acetamide [compound 7c]

Yield: $73.3 \%$; M. P. $210-212{ }^{\circ} \mathrm{C}$; Anal. Cal. for $\mathrm{C}_{20} \mathrm{H}_{19} \mathrm{~N}_{3} \mathrm{O}_{3} \mathrm{~S}_{3}$ : C, 53.91; H, 4.30; N, 9.43; O, 10.77; S, 21.59 \%; found: C, 53.93; H, 4.32; N, 9.44; 0, 10.71; S, 21.60\%.

\section{FT-IR spectroscopy}

FT-IR (vmax): 3266 (N-H strech.), 3035 (aromatic C-H strech.), 2969(asym. aliphatic C-H strech.), 2862 (sym. aliphatic C-H strech.), 1670-2000 (overtone for substitution on aromatic ring), 1712 (cyclic $\mathrm{C}=0$ strech.), 1660 (amide $\mathrm{C}=0$ stretch.), 1599 (phenyl ring stretch.), 1579 ( $\mathrm{N}-\mathrm{H}$ bending), 1515 (phenyl C-H out of plane bending), $1464\left(\mathrm{CH}_{2}\right.$ bending), 1455 (asym $\mathrm{CH}_{3}$ bending), 1387 (sym. $\mathrm{CH}_{3}$ bending), 1315 (C-N Strech), 1250 (asym. C-O strech.), 1038 (sym. C-0 stretch.), 694 (C-S stretch) $\mathrm{cm}^{-1}$.

\section{${ }^{1}$ H-NMR spectroscopy}

${ }^{1} \mathrm{H}-\mathrm{NMR}\left(\mathrm{CDCl}_{3}\right)(\delta, \mathrm{ppm}): 7.94(\mathrm{~s}, 1 \mathrm{H},-\mathrm{CONH}-), 7.89-7.63(\mathrm{~m}, 3 \mathrm{H}$, benzthiazole ring protons), 7.35-6.86 (m, $4 \mathrm{H}$, phenyl ring protons), $5.91(\mathrm{~s}, 1 \mathrm{H}$, thiazole ring protons at phenyl linkage), $4.35(\mathrm{~s}, 2 \mathrm{H}$,$\left.\mathrm{SCH}_{2}-\right), 3.95-3.82\left(\mathrm{~s}, 2 \mathrm{H}\right.$, thiazole ring protons), $2.34\left(\mathrm{~s}, 3 \mathrm{H}, \mathrm{CH}_{3}\right.$ at benzthiazole linkage).

\section{${ }^{13} \mathrm{C}$-NMR spectroscopy}

${ }^{13} \mathrm{C}-\mathrm{NMR}\left(\mathrm{CDCl}_{3}\right)(\delta, \mathrm{ppm}): 170.9$ (C=0, amide carbon), 168.5 ( $\mathrm{C}=0$ thiazole ring carbon), $163.8(\mathrm{C}=\mathrm{N}$ bezthiazole ring carbon at sulfur linkage), 151.9 (C-N benzthiazole ring carbon), 159.2 (phenyl ring carbon at methoxy linkage), 134.6-136.2 (bezthiazole ring carbons at sulfur and methyl linkage), 131.5 (phenyl ring carbon at thiazole linkage), 121.1-126.3 (benzthiazole ring carbons at proton linkage), 114.2-125.9 (phenyl ring carbons), 58.3 (thiazole ring carbon at phenyl linkage), 41.9 (-S- $\mathrm{CH}_{2}-\mathrm{CONH}-$ ), 36.8 (thiazole ring carbon at- $\mathrm{C}=0$ linkage), 24.3 (methyl carbon at benzthiazole ring).

\section{Mass spectroscopy}

Molecular ion peak demonstrated on mass spectrum and reported m/e (ESI): $446\left(\mathrm{M}^{+}\right)$.

\section{(D) 2-(5-methylbenzo[d]thiazol-2-ylthio)-N-(2-(3-nitrophenyl)-} 4-oxothiazolidin-3-yl) acetamide [compound 7d]

Yield: $76.2 \%$; M. P. $276-278{ }^{\circ} \mathrm{C}$; Anal. Cal. for $\mathrm{C}_{19} \mathrm{H}_{16} \mathrm{~N}_{4} \mathrm{O}_{4} \mathrm{~S}_{3}$ : C, 49.55; H, 3.50; N, 12.17; 0, 13.90; S, $20.89 \%$; found: C, 49.53; H, 3.48; N, 12.14; O, 13.95; S, $20.91 \%$.

\section{FT-IR spectroscopy}

FT-IR (vmax): 3258 (N-H strech.), 3038 (aromatic C-H strech.), 2972(asym. aliphatic C-H strech.), 2865 (sym. aliphatic C-H strech.), 1670-2000 (overtone for substitution on aromatic ring), 1714 (cyclic C=0 strech.), 1658 (amide $\mathrm{C}=0$ stretch.), 1597 (phenyl ring stretch.), 1575 ( $\mathrm{N}-\mathrm{H}$ bending), 1538 (asym. $\mathrm{NO}_{2}$ strech), 1516 (phenyl C-H out of plane bending), $1466\left(\mathrm{CH}_{2}\right.$ bending), 1453 (asym $\mathrm{CH}_{3}$ bending), 1389 (sym. $\mathrm{CH}_{3}$ bending), 1334 (sym $\mathrm{NO}_{2}$ bending), 1312 (C-N Strech), 696 (C-S stretch) $\mathrm{cm}^{-1}$. FT-IR image of the synthesized compound is shown in fig. 2.

\section{${ }^{1} \mathrm{H}$-NMR spectroscopy}

${ }^{1} \mathrm{H}-\mathrm{NMR}\left(\mathrm{CDCl}_{3}\right)(\delta, \mathrm{ppm}): 8.10(\mathrm{~s}, 1 \mathrm{H},-\mathrm{CONH}-), 8.06-7.87(\mathrm{~m}, 4 \mathrm{H}$, phenyl ring protons), 7.81-7.33 ( $\mathrm{m}, 3 \mathrm{H}$, benzthiazole ring protons), $5.92(\mathrm{~s}, 1 \mathrm{H}$, thiazole ring protons at phenyl linkage), $4.35(\mathrm{~s}, 2 \mathrm{H},-$ $\mathrm{SCH}_{2}-$ ), 3.95-3.83 (s, $2 \mathrm{H}$, thiazole ring protons), $2.34\left(\mathrm{~s}, 3 \mathrm{H}, \mathrm{CH}_{3}\right.$ at benzthiazole linkage). ${ }^{1} H-N M R$ image of the synthesized compound is shown in fig. 3. 


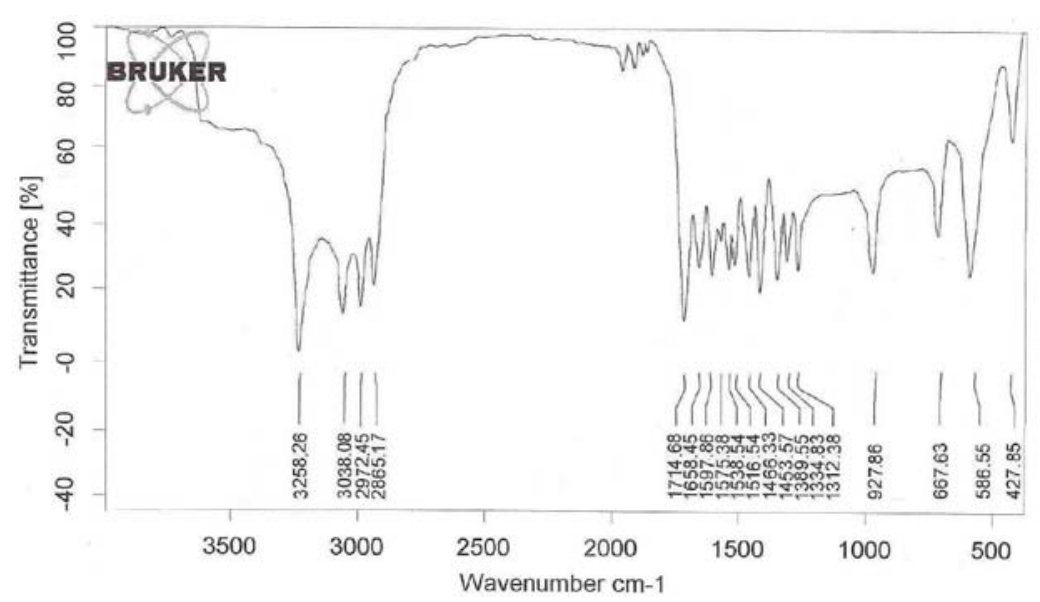

Fig. 2: FT-IR image of synthesised compound 7d

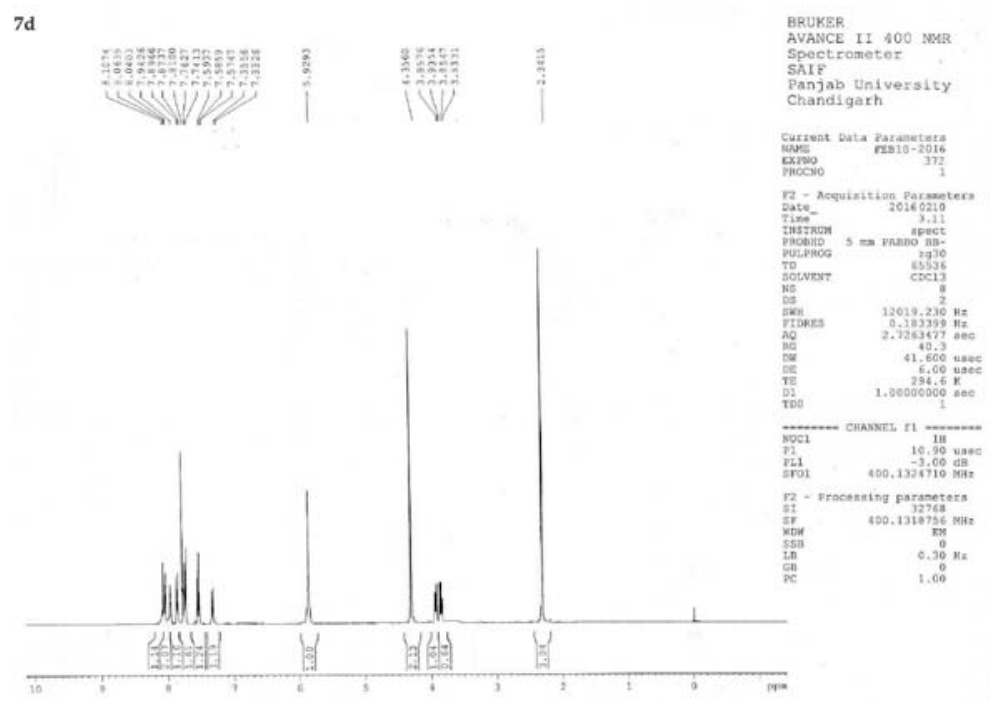

Fig. 3: ${ }^{1} H-N M R$ image of synthesised compound 7d

\section{${ }^{13}$ C-NMR spectroscopy}

${ }^{13} \mathrm{C}-\mathrm{NMR}\left(\mathrm{CDCl}_{3}\right)(\delta, \mathrm{ppm}): 171.2(\mathrm{C}=0$, amide carbon $), 167.9(\mathrm{C}=0$ thiazole ring carbon), $164.2(\mathrm{C}=\mathrm{N}$ bezthiazole ring carbon at sulfur linkage), 152.3 (C-N benzthiazole ring carbon), 147.2 (phenyl ring carbon at nitro linkage), 145.3 (phenyl ring carbon at thiazole linkage), 134.3-135.5 (bezthiazole ring carbons at sulfur and methyl linkage), 121.4-126.7 (benzthiazole ring carbons at proton linkage), 121.1-129.2 (phenyl ring carbons), 57.6 (thiazole ring carbon at phenyl linkage), 40.5 (-S- $\mathrm{CH}_{2}-\mathrm{CONH}-$ ), 35.9 (thiazole ring carbon at$\mathrm{C}=0$ linkage), 23.7 (methyl carbon at benzthiazole ring).

\section{Mass spectroscopy}

Molecular ion peak demonstrated on mass spectrum and reported $\mathrm{m} / \mathrm{e}(\mathrm{ESI}): 461\left(\mathrm{M}^{+}\right)$.

(E) 2-(5-nitrobenzo[d]thiazol-2-ylthio)-N-(4-oxo-2phenylthiazolidin-3-yl)acetamide [compound 7e]

Yield: $59.5 \%$; M. P. $165-167{ }^{\circ} \mathrm{C}$; Anal. Cal. for $\mathrm{C}_{18} \mathrm{H}_{14} \mathrm{~N}_{4} \mathrm{O}_{4} \mathrm{~S}_{3}$ : C, 48.42; H, 3.16; N, 12.55; 0, 14.33; S, 21.54\%; found: C, 48.39; H, 3.13; $\mathrm{N}, 12.59 ; 0,14.34 ; \mathrm{S}, 21.55 \%$.

\section{FT-IR spectroscopy}

FT-IR (vmax): 3263 (N-H strech.), 3032 (aromatic C-H strech.), 2964 (asym. aliphatic C-H strech.), 2862 (sym. aliphatic C-H strech.), 1670-2000 (overtone for substitution on aromatic ring), 1714(cyclic
C=0 strech.), 1665 (amide $\mathrm{C}=0$ stretch.), 1602 (phenyl ring stretch.) 1581 ( $\mathrm{N}-\mathrm{H}$ bending), 1536 (asym. $\mathrm{N}=\mathrm{O}$ strech), 1513 (phenyl C-H out of plane bending), 1467 ( $\mathrm{CH}_{2}$ bending), 1342 (sym. $\mathrm{N}=0$ strech), 1318 (C-N Strech), 687 (C-S stretch) $\mathrm{cm}^{-1}$.

\section{H-NMR spectroscopy}

${ }^{1} \mathrm{H}-\mathrm{NMR}\left(\mathrm{CDCl}_{3}\right)(\delta, \mathrm{ppm}): 8.66(\mathrm{~s}, 1 \mathrm{H},-\mathrm{CONH}-), 8.35-7.95(\mathrm{~m}, 3 \mathrm{H}$ benzthiazole ring protons), 7.35-7.18 (m, $5 \mathrm{H}$, phenyl ring protons), $5.92(\mathrm{~s}, 1 \mathrm{H}$, thiazole ring protons at phenyl linkage), $4.35(\mathrm{~s}, 2 \mathrm{H}$,$\mathrm{SCH}_{2}-$ ), 3.95-3.82 (s, $2 \mathrm{H}$, thiazole ring protons).

\section{${ }^{13} \mathrm{C}$-NMR spectroscopy}

${ }^{13} \mathrm{C}-\mathrm{NMR}\left(\mathrm{CDCl}_{3}\right)(\delta, \mathrm{ppm}): 172.1(\mathrm{C}=0$, amide carbon), $169.3(\mathrm{C}=0$ thiazole ring carbon), $165.8(\mathrm{C}=\mathrm{N}$ bezthiazole ring carbon at sulfur linkage), 154.4 (C-N benzthiazole ring carbon), 140.1 (phenyl ring carbon at thiazole linkage), 141.1-145.2 (bezthiazole ring carbons at sulfur and nitro linkage), 126.8-129.3 (phenyl ring carbons), 117.1122.2 (benzthiazole ring carbons at proton linkage), 58.7 (thiazole ring carbon at phenyl linkage), $41.6\left(-\mathrm{S}-\mathrm{CH}_{2}-\mathrm{CONH}-\right), 37.1$ (thiazole ring carbon at $-\mathrm{C}=0$ linkage).

\section{Mass spectroscopy}

Molecular ion peak demonstrated on mass spectrum and reported m/e (ESI): $447\left(\mathrm{M}^{+}\right)$.

(F) N-(2-(4-hydroxyphenyl)-4-oxothiazolidin-3-yl)-2-(5nitrobenzo[d]thiazol-2-ylthio) acetamide [compound 7f] 
Yield: $63.2 \%$; M. P. $184-186{ }^{\circ} \mathrm{C}$; Anal. Cal. for $\mathrm{C}_{18} \mathrm{H}_{14} \mathrm{~N}_{4} \mathrm{O}_{5} \mathrm{~S}_{3}$ : C 46.74; H, 3.05; N, 12.11; 0, 17.30; S, 20.80\%; found: C, 46.76; H, 3.08; $\mathrm{N}, 12.06 ; 0,17.34 ; \mathrm{S}, 20.76 \%$

\section{FT-IR spectroscopy}

FT-IR (vmax): 3468 (O-H stretch.), 3274 (N-H strech.), 3038 (Aromatic C-H strech.), 2969 (asym. aliphatic C-H strech.), 2867 (sym. aliphatic C-H strech.), 1670-2000 (overtone for substitution on aromatic ring), 1716 (cyclic $\mathrm{C}=0$ strech.), 1665 (amide $\mathrm{C}=0$ stretch.), 1601 (phenyl ring stretch.), 1578 ( $\mathrm{N}-\mathrm{H}$ bending), 1538 (asym. N=0 strech), 1509 (phenyl C$\mathrm{H}$ out of plane bending), $1469\left(\mathrm{CH}_{2}\right.$ bending), 1342 (sym. $\mathrm{N}=0$ strech), 1319 (C-N Strech), 689 (C-S stretch) $\mathrm{cm}^{-1}$.

\section{${ }^{1} \mathrm{H}$-NMR spectroscopy}

${ }^{1} \mathrm{H}-\mathrm{NMR}\left(\mathrm{CDCl}_{3}\right)(\delta, \mathrm{ppm}): 8.66(\mathrm{~s}, 1 \mathrm{H},-\mathrm{CONH}-), 8.35-7.95(\mathrm{~m}, 3 \mathrm{H}$, benzthiazole ring protons), 7.79-6.63 ( $\mathrm{m}, 4 \mathrm{H}$, phenyl ring protons), 5.91 $\left(\mathrm{s}, 1 \mathrm{H}\right.$, thiazole ring protons at phenyl linkage), $5.35\left(\mathrm{~s}, 1 \mathrm{H},-\mathrm{C}_{6} \mathrm{H}_{4}-\mathrm{OH}\right)$, $4.35\left(\mathrm{~s}, 2 \mathrm{H},-\mathrm{SCH}_{2}-\right), 3.95-3.83(\mathrm{~s}, 2 \mathrm{H}$, thiazole ring protons).

\section{${ }^{13} \mathrm{C}$-NMR spectroscopy}

${ }^{13} \mathrm{C}$-NMR $\left(\mathrm{CDCl}_{3}\right)(\delta, \mathrm{ppm}): 171.1(\mathrm{C}=0$, amide carbon $), 170.4(\mathrm{C}=0$ thiazole ring carbon), $164.2(\mathrm{C}=\mathrm{N}$ bezthiazole ring carbon at sulfur linkage), 154.6 (C-N benzthiazole ring carbon), 156.3 (phenyl ring carbon at hydroxyl linkage), 141.2-145.4 (bezthiazole ring carbons at sulfur and nitro linkage), 131.3 (phenyl ring carbon at thiazole linkage), 124.2-130.7 (phenyl ring carbons), 117.3-122.9 (benzthiazole ring carbons at proton linkage), 58.4 (thiazole ring carbon at phenyl linkage), $41.8\left(-\mathrm{S}-\mathrm{CH}_{2}-\mathrm{CONH}-\right.$ ), 37.2 (thiazole ring carbon at- $\mathrm{C}=0$ linkage).

\section{Mass spectroscopy}

Molecular ion peak demonstrated on mass spectrum and reported m/e (ESI): $463\left(\mathrm{M}^{+}\right)$.

(G) N-(2-(4-methoxyphenyl)-4-oxothiazolidin-3-yl)-2-(5-nitrobenzo[d]thiazol-2-ylthio) acetamide [compound 7g]

Yield: $69.1 \%$; M. P. 207-209 ${ }^{\circ} \mathrm{C}$; Anal. Cal. for $\mathrm{C}_{19} \mathrm{H}_{16} \mathrm{~N}_{4} \mathrm{O}_{5} \mathrm{~S}_{3}$ : C, 47.89; H, 3.38; N, 11.76; O, 16.79; S, 20.19\%; found: C, 47.92; H, 3.34; $\mathrm{N}, 11.73 ; 0,16.81 ; \mathrm{S}, 20.21 \%$.

\section{FT-IR spectroscopy}

FT-IR (vmax): 3268 (N-H strech.), 3037 (aromatic C-H strech.), 2972 (asym. aliphatic C-H strech.), 2865 (sym. aliphatic C-H strech.), 1670-2000 (overtone for substitution on aromatic ring), 1713 (cyclic $\mathrm{C}=0$ strech.), 1663 (amide $\mathrm{C}=0$ stretch.), 1602 (phenyl ring stretch.), 1577 ( $\mathrm{N}-\mathrm{H}$ bending), 1539 (asym. $\mathrm{N}=\mathrm{O}$ strech), 1512 (phenyl C-H out of plane bending), $1463\left(\mathrm{CH}_{2}\right.$ bending), 1452 (asym $\mathrm{CH}_{3}$ bending), 1384 (sym. $\mathrm{CH}_{3}$ bending), 1344 (Sym. $\mathrm{N}=\mathrm{O}$ strech), 1316 (C-N Strech), 1254 (asym. C-O strech.), 1040 (sym. C-O stretch.), 696 (C-S stretch) $\mathrm{cm}^{-1}$.

\section{${ }^{1}$ H-NMR spectroscopy}

${ }^{1} \mathrm{H}-\mathrm{NMR}\left(\mathrm{CDCl}_{3}\right)(\delta, \mathrm{ppm}): 8.62(\mathrm{~s}, 1 \mathrm{H},-\mathrm{CONH}-), 8.35-7.95(\mathrm{~m}, 3 \mathrm{H}$, benzthiazole ring protons), 7.84-6.85 (m, $4 \mathrm{H}$, phenyl ring protons), $5.92(\mathrm{~s}, 1 \mathrm{H}$, thiazole ring protons at phenyl linkage), $4.35(\mathrm{~s}, 2 \mathrm{H}$,$\mathrm{SCH}_{2}-$ ), 3.95-3.83 (s, $2 \mathrm{H}$, thiazole ring protons).

\section{${ }^{13} \mathrm{C}$-NMR spectroscopy}

${ }^{13} \mathrm{C}$-NMR $\left(\mathrm{CDCl}_{3}\right)(\delta, \mathrm{ppm}): 171.4(\mathrm{C}=0$, amide carbon), $169.2(\mathrm{C}=0$ thiazole ring carbon), 164.1 ( $\mathrm{C}=\mathrm{N}$ bezthiazole ring carbon at sulfur linkage), 154.1 (C-N benzthiazole ring carbon), 159.5 (phenyl ring carbon at methoxy linkage), 141.3-145.4 (bezthiazole ring carbons at sulfur and nitro linkage), 131.2 (phenyl ring carbon at thiazole linkage), 124.6-129.3 (phenyl ring carbons), 117.3-122.9 (benzthiazole ring carbons at proton linkage), 57.9 (thiazole ring carbon at phenyl linkage), $41.4\left(-\mathrm{S}-\mathrm{CH}_{2}-\mathrm{CONH}-\right), 36.4$ (thiazole ring carbon at- $\mathrm{C}=0$ linkage).

\section{Mass spectroscopy}

Molecular ion peak demonstrated on mass spectrum and reported m/e (ESI): $477\left(\mathrm{M}^{+}\right)$.

(H) 2-(5-nitrobenzo[d]thiazol-2-ylthio)-N-(2-(3-nitrophenyl)-4oxothiazolidin-3-yl) acetamide [compound $7 \mathrm{~h}$ ]
Yield: $74.8 \%$; M. P. $273-275{ }^{\circ} \mathrm{C}$; Anal. Cal. for $\mathrm{C}_{18} \mathrm{H}_{13} \mathrm{~N}_{5} \mathrm{O}_{6} \mathrm{~S}_{3}$ : C, 43.98; H, 2.67; N, 14.25; O, 19.53; S, $19.57 \%$; found: C, 43.96; $\mathrm{H}$, $2.65 ; \mathrm{N}, 14.23 ; 0,19.56 ; \mathrm{S}, 19.60 \%$.

\section{FT-IR spectroscopy}

FT-IR (vmax): 3261 (N-H strech.), 3040 (aromatic C-H strech.), 2975 (asym. aliphatic C-H strech.), 2868 (sym. aliphatic C-H strech.), 1670-2000 (overtone for substitution on aromatic ring), 1716 (cyclic $\mathrm{C}=0$ strech.), 1661 (amide $\mathrm{C}=0$ stretch.), 1599 (phenyl ring stretch.), 1577 ( $\mathrm{N}-\mathrm{H}$ bending), 1540 (asym. $\mathrm{NO}_{2}$ strech), 1514 (phenyl C-H out of plane bending), 1468 ( $\mathrm{CH}_{2}$ bending), 1337 (sym. $\mathrm{NO}_{2}$ bending), 1314 (C-N Strech), 694 (C-S stretch) $\mathrm{cm}^{-1}$.

\section{${ }^{1} \mathrm{H}-\mathrm{NMR}$ spectroscopy}

${ }^{1} \mathrm{H}-\mathrm{NMR}\left(\mathrm{CDCl}_{3}\right)(\delta, \mathrm{ppm}): 8.62(\mathrm{~s}, 1 \mathrm{H},-\mathrm{CONH}-), 8.34-7.96(\mathrm{~m}, 3 \mathrm{H}$, benzthiazole ring protons), 7.75-7.56 (m, $4 \mathrm{H}$, phenyl ring protons), $5.93(\mathrm{~s}, 1 \mathrm{H}$, thiazole ring protons at phenyl linkage), $4.35(\mathrm{~s}, 2 \mathrm{H},-$ $\mathrm{SCH}_{2}-$ ), 3.95-3.83(s, $2 \mathrm{H}$, thiazole ring protons).

\section{${ }^{13} \mathrm{C}$-NMR spectroscopy}

${ }^{13} \mathrm{C}$-NMR $\left(\mathrm{CDCl}_{3}\right)(\delta, \mathrm{ppm}): 171.9(\mathrm{C}=0$, amide carbon), $168.8(\mathrm{C}=0$ thiazole ring carbon), $164.9(\mathrm{C}=\mathrm{N}$ bezthiazole ring carbon at sulfur linkage), 155.2 (C-N benzthiazole ring carbon), 147.8 (phenyl ring carbon at nitro linkage), 145.9 (phenyl ring carbon at thiazole linkage), 134.3-135.5 (bezthiazole ring carbons at sulfur and nitro linkage), 141.6-145.9 (benzthiazole ring carbons at proton linkage), 121.6-129.8 (phenyl ring carbons), 58.1 (thiazole ring carbon at phenyl linkage), $41.3\left(-\mathrm{S}^{-\mathrm{CH}_{2}}-\mathrm{CONH}-\right), 36.5$ (thiazole ring carbon at$\mathrm{C}=\mathrm{O}$ linkage).

\section{Mass spectroscopy}

Molecular ion peak demonstrated on mass spectrum and reported m/e (ESI): $492\left(\mathrm{M}^{+}\right)$.

\section{(I) 2-(6-nitrobenzo[d]thiazol-2-ylthio)-N-(4-oxo-2- phenylthiazolidin-3-yl)acetamide [compound 7i]}

Yield: $56.8 \%$; M. P. $161-163{ }^{\circ} \mathrm{C}$; Anal. Cal. for $\mathrm{C}_{18} \mathrm{H}_{14} \mathrm{~N}_{4} \mathrm{O}_{4} \mathrm{~S}_{3}$ : C, 48.42; H, 3.16; N, 12.55 ; O, 14.33; S, 21.54\%; found: C, 48.45; H, 3.12; $\mathrm{N}, 12.52 ; 0,14.36 ; \mathrm{S}, 21.55 \%$.

\section{FT-IR spectroscopy}

FT-IR (vmax): 3267 (N-H strech.), 3036 (aromatic C-H strech.), 2969 (asym. aliphatic C-H strech.), 2868 (sym. aliphatic C-H strech.), 1670-2000 (overtone for substitution on aromatic ring), 1716 (cyclic $\mathrm{C}=0$ strech.), 1668 (amide $\mathrm{C}=0$ stretch.), 1605 (phenyl ring stretch.), 1584 ( $\mathrm{N}-\mathrm{H}$ bending), 1539 (asym. $\mathrm{N}=\mathrm{O}$ strech), 1516 (phenyl C-H out of plane bending), $1469\left(\mathrm{CH}_{2}\right.$ bending), 1345 (sym. $\mathrm{N}=0$ strech), 1319 (C-N Strech), 694 (C-S stretch) $\mathrm{cm}^{-1}$.

\section{${ }^{1} \mathrm{H}-\mathrm{NMR}$ spectroscopy}

${ }^{1} \mathrm{H}-\mathrm{NMR}\left(\mathrm{CDCl}_{3}\right)(\delta, \mathrm{ppm}): 9.17(\mathrm{~s}, 1 \mathrm{H},-\mathrm{CONH}-), 8.34-8.25(\mathrm{~m}, 3 \mathrm{H}$, benzthiazole ring protons), 7.37-7.18 (m, $5 \mathrm{H}$, phenyl ring protons), $5.93(\mathrm{~s}, 1 \mathrm{H}$, thiazole ring protons at phenyl linkage), $4.35(\mathrm{~s}, 2 \mathrm{H}$,$\mathrm{SCH}_{2}-$ ), 3.95-3.83(s, $2 \mathrm{H}$, thiazole ring protons).

\section{${ }^{13} \mathrm{C}$-NMR spectroscopy}

${ }^{13} \mathrm{C}-\mathrm{NMR}\left(\mathrm{CDCl}_{3}\right)(\delta, \mathrm{ppm}): 173.1(\mathrm{C}=0$, amide carbon $), 169.9(\mathrm{C}=0$ thiazole ring carbon), $166.5(\mathrm{C}=\mathrm{N}$ bezthiazole ring carbon at sulfur linkage), 159.6 (C-N benzthiazole ring carbon), 140.8 (phenyl ring carbon at thiazole linkage), 142.4-146.6 (bezthiazole ring carbons at sulfur and nitro linkage), 125.3-128.9 (phenyl ring carbons), 118.3123.5 (benzthiazole ring carbons at proton linkage), 59.2 (thiazole ring carbon at phenyl linkage), $43.2\left(-\mathrm{S}-\mathrm{CH}_{2}-\mathrm{CONH}-\right.$ ), 36.8 (thiazole ring carbon at- $\mathrm{C}=0$ linkage).

\section{Mass spectroscopy}

Molecular ion peak demonstrated on mass spectrum and reported m/e (ESI): $447\left(\mathrm{M}^{+}\right)$.

(J) N-(2-(4-hydroxyphenyl)-4-oxothiazolidin-3-yl)-2-(6nitrobenzo[d]thiazol-2-ylthio) acetamide [compound $7 \mathrm{j}]$ 
Yield: $61.3 \%$; M. P. $179-181{ }^{\circ} \mathrm{C}$; Anal. Cal. for $\mathrm{C}_{18} \mathrm{H}_{14} \mathrm{~N}_{4} \mathrm{O}_{5} \mathrm{~S}_{3}$ : C, 46.74; H, 3.05; N, 12.11; 0, 17.30; S, $20.80 \%$; found: C, 46.76; H, $3.01 ; \mathrm{N}, 12.08 ; 0,17.33 ; \mathrm{S}, 20.82 \%$.

\section{FT-IR spectroscopy}

FT-IR (vmax): 3472 (O-H stretch.), 3278 (N-H strech.), 3042 (aromatic C-H strech.), 2973 (asym. aliphatic C-H strech.), 2869 (sym. aliphatic C-H strech.), 1670-2000 (overtone for substitution on aromatic ring), 1714 (cyclic $\mathrm{C}=0$ strech.), 1667 (amide $\mathrm{C}=0$ stretch.), 1599 (phenyl ring stretch.), 1581 (N-H bending), 1541 (asym. $\mathrm{N}=\mathrm{O}$ strech), 1511 (phenyl $\mathrm{C}-\mathrm{H}$ out of plane bending), 1466 ( $\mathrm{CH}_{2}$ bending), 1345 (sym. N=O strech), 1316 (C-N Strech), 692 (C-S stretch) $\mathrm{cm}^{-1}$.

\section{${ }^{1}$ H-NMR spectroscopy}

${ }^{1} \mathrm{H}-\mathrm{NMR}\left(\mathrm{CDCl}_{3}\right)(\delta, \mathrm{ppm}): 9.16(\mathrm{~s}, 1 \mathrm{H},-\mathrm{CONH}-), 8.34-7.96(\mathrm{~m}, 3 \mathrm{H}$, benzthiazole ring protons), 7.77-6.63 (m, $4 \mathrm{H}$, phenyl ring protons), $5.93(\mathrm{~s}, 1 \mathrm{H}$, thiazole ring protons at phenyl linkage), $5.35(\mathrm{~s}, 1 \mathrm{H},-$ $\left.\mathrm{C}_{6} \mathrm{H}_{4}-\mathrm{OH}\right), 4.35\left(\mathrm{~s}, 2 \mathrm{H},-\mathrm{SCH}_{2}-\right), 3.95-3.83(\mathrm{~s}, 2 \mathrm{H}$, thiazole ring protons).

\section{${ }^{13}$ C-NMR spectroscopy}

${ }^{13} \mathrm{C}$-NMR $\left(\mathrm{CDCl}_{3}\right)(\delta, \mathrm{ppm}): 173.3(\mathrm{C}=0$, amide carbon $), 172.2(\mathrm{C}=0$ thiazole ring carbon), 165.2 ( $\mathrm{C}=\mathrm{N}$ bezthiazole ring carbon at sulfur linkage), 160.2 (C-N benzthiazole ring carbon), 157.7 (phenyl ring carbon at hydroxyl linkage), 143.6-146.8 (bezthiazole ring carbons at sulfur and nitro linkage), 132.4 (phenyl ring carbon at thiazole linkage), 125.7-130.9 (phenyl ring carbons), 118.6-123.8 (benzthiazole ring carbons at proton linkage), 59.3 (thiazole ring carbon at phenyl linkage), 43.2 (-S- $\left.\mathrm{CH}_{2}-\mathrm{CONH}-\right), 38.6$ (thiazole ring carbon at $-\mathrm{C}=\mathrm{O}$ linkage).

\section{Mass spectroscopy}

Molecular ion peak demonstrated on mass spectrum and reported m/e (ESI): $463\left(\mathrm{M}^{+}\right)$.

\section{(K) N-(2-(4-methoxyphenyl)-4-oxothiazolidin-3-yl)-2-(6-nitro- benzo[d]thiazol-2-ylthio) acetamide [compound $7 \mathrm{k}$ ]}

Yield: $67.7 \%$; M. P. $202-204{ }^{\circ} \mathrm{C}$; Anal. Cal. for $\mathrm{C}_{19} \mathrm{H}_{16} \mathrm{~N}_{4} \mathrm{O}_{5} \mathrm{~S}_{3}$ : C, 47.89; H, 3.38; N, 11.76; 0, 16.79; S, $20.19 \%$; found: C, 47.92; H, 3.34; N, 11.73; O, 16.81; S, $20.21 \%$.

\section{FT-IR spectroscopy}

FT-IR (vmax): 3271 (N-H strech.), 3040 (aromatic C-H strech.), 2976 (asym. aliphatic C-H strech.), 2868 (sym. aliphatic C-H strech.), 1670-2000 (overtone for substitution on aromatic ring), 1716 (cyclic $\mathrm{C}=0$ strech.), 1666 (amide $\mathrm{C}=0$ stretch.), 1604 (phenyl ring stretch.), 1577 ( $\mathrm{N}-\mathrm{H}$ bending), 1542 (asym. $\mathrm{N}=\mathrm{O}$ strech), 1514 (phenyl C-H out of plane bending), 1467 ( $\mathrm{CH}_{2}$ bending), 1456 (asym $\mathrm{CH}_{3}$ bending), 1386 (sym. $\mathrm{CH}_{3}$ bending), 1347 (sym. $\mathrm{N}=\mathrm{O}$ strech), 1316 (C-N strech), 1253 (asym. C-O strech.), 1041 (sym. C-O stretch.), 693 (C-S stretch) $\mathrm{cm}^{-1}$.

\section{H-NMR spectroscopy}

${ }^{1} \mathrm{H}-\mathrm{NMR}\left(\mathrm{CDCl}_{3}\right)(\delta, \mathrm{ppm}): 9.16(\mathrm{~s}, 1 \mathrm{H},-\mathrm{CONH}-), 8.34-7.96(\mathrm{~m}, 3 \mathrm{H}$, benzthiazole ring protons), 7.86-6.66 (m, $4 \mathrm{H}$, phenyl ring protons), $5.92(\mathrm{~s}, 1 \mathrm{H}$, thiazole ring protons at phenyl linkage), $4.35(\mathrm{~s}, 2 \mathrm{H}$,$\mathrm{SCH}_{2}-$ ), 3.95-3.83 (s, $2 \mathrm{H}$, thiazole ring protons).

\section{${ }^{13} \mathrm{C}$-NMR spectroscopy}

${ }^{13} \mathrm{C}$-NMR $\left(\mathrm{CDCl}_{3}\right)(\delta, \mathrm{ppm}): 172.5(\mathrm{C}=0$, amide carbon), 169.9 (C=0 thiazole ring carbon), 165.5 ( $\mathrm{C}=\mathrm{N}$ bezthiazole ring carbon at sulfur linkage), 161.8 (C-N benzthiazole ring carbon), 158.8 (phenyl ring carbon at methoxy linkage), 141.9-146.2 (bezthiazole ring carbons at sulfur and nitro linkage), 132.1 (phenyl ring carbon at thiazole linkage), 124.1-128.9 (phenyl ring carbons), 118.1-123.2 (benzthiazole ring carbons at proton linkage), 58.4 (thiazole ring carbon at phenyl linkage), 42.1 (-S- $\mathrm{CH}_{2}-\mathrm{CONH}-$ ), 37.3 (thiazole ring carbon at $-\mathrm{C}=\mathrm{O}$ linkage).

\section{Mass spectroscopy}

Molecular ion peak demonstrated on mass spectrum and reported m/e (ESI): $477\left(\mathrm{M}^{+}\right)$.

\section{(L) 2-(6-nitrobenzo[d]thiazol-2-ylthio)-N-(2-(3-nitrophenyl)-4- oxothiazolidin-3-yl) acetamide [compound 7l]}

Yield: 71.9 \%; M. P. $269-271{ }^{\circ} \mathrm{C}$; Anal. Cal. for $\mathrm{C}_{18} \mathrm{H}_{13} \mathrm{~N}_{5} \mathrm{O}_{6} \mathrm{~S}_{3}$ : C, 43.98; H, 2.67; N, 14.25; 0, 19.53; S, $19.57 \%$; found: C, 44.01; $\mathrm{H}$, 2.64; N, 14.21; O, 19.56; S, $19.58 \%$.

\section{FT-IR spectroscopy}

FT-IR (vmax): 3265 (N-H strech.), 3043 (aromatic C-H strech.), 2978 (asym. aliphatic C-H strech.), 2873 (sym. aliphatic C-H strech.), 1670-2000 (overtone for substitution on aromatic ring), 1715 (cyclic C=0 strech.), 1664 (amide $\mathrm{C}=0$ stretch.), 1601 (phenyl ring stretch.), 1575 ( $\mathrm{N}-\mathrm{H}$ bending), 1543 (asym. $\mathrm{NO}_{2}$ strech), 1515 (phenyl C-H out of plane bending), 1466 ( $\mathrm{CH}_{2}$ bending), 1339 (sym $\mathrm{NO}_{2}$ bending), 1317 (C-N Strech), 694 (C-S stretch) $\mathrm{cm}^{-1}$.

\section{H-NMR spectroscopy}

${ }^{1} \mathrm{H}-\mathrm{NMR}\left(\mathrm{CDCl}_{3}\right)(\delta, \mathrm{ppm}): 9.16(\mathrm{~s}, 1 \mathrm{H},-\mathrm{CONH}-), 8.34-7.96(\mathrm{~m}, 3 \mathrm{H}$, benzthiazole ring protons), 7.75-7.56 (m, $4 \mathrm{H}$, phenyl ring protons), $5.93(\mathrm{~s}, 1 \mathrm{H}$, thiazole ring protons at phenyl linkage), $4.35(\mathrm{~s}, 2 \mathrm{H}$,$\mathrm{SCH}_{2}-$ ), 3.95-3.83 (s, $2 \mathrm{H}$, thiazole ring protons).

\section{${ }^{13} \mathrm{C}$-NMR spectroscopy}

${ }^{13} \mathrm{C}-\mathrm{NMR}\left(\mathrm{CDCl}_{3}\right)(\delta, \mathrm{ppm}): 172.7(\mathrm{C}=0$, amide carbon), $170.1(\mathrm{C}=0$ thiazole ring carbon), $164.7(\mathrm{C}=\mathrm{N}$ bezthiazole ring carbon at sulfur linkage), 161.4 (C-N benzthiazole ring carbon), 149.7 (phenyl ring carbon at nitro linkage), 146.6 (phenyl ring carbon at thiazole linkage), 135.9-137.1 (bezthiazole ring carbons at sulfur and nitro linkage), 142.9-146.2 (benzthiazole ring carbons at proton linkage), 121.9-129.4 (phenyl ring carbons), 59.4 (thiazole ring carbon at phenyl linkage), 43.1 (-S-CH 2 -CONH-), 38.3 (thiazole ring carbon at$\mathrm{C}=0$ linkage).

\section{Mass spectroscopy}

Molecular ion peak demonstrated on mass spectrum and reported m/e (ESI): $492\left(M^{+}\right)$.

\section{Evaluation of antidiabetic activity in alloxan-induced diabetic rats}

The LD50 values of the synthesised compounds were estimated to be in the range of $300-3000 \mathrm{mg} / \mathrm{kg}$ b.w. Alloxan induces diabetes through rapid depletion of $\beta$-cells which ultimately results to reduce the insulin release. All the results summarised in table 2 revealed that most of the synthesised compounds exhibited antidiabetic response at the end of ten-day of the experimental period. It has been found that oral administration of synthesised compounds $7 \mathrm{~d}$, $7 \mathrm{~g}, 7 \mathrm{~h}$ and $7 \mathrm{l}$ caused a more significant reduction in blood glucose than other compounds in diabetic rats. However, the compound $7 \mathrm{~d}$ at $350 \mathrm{mg} / \mathrm{kg}$ b.w. exerted maximum glucose lowering effects whereas 7c showed minimum glucose lowering effects.

\section{DISCUSSION}

This study was undertaken; mainly to assess the protective effect of benzothiazole derivatives against alloxan-induced diabetes in experimental rats. Alloxan is selectively toxic to pancreatic $\beta$ cells that produce insulin due to the accumulation of alloxan through the GLUT $_{2}$ transporter. Though alloxan by itself is not toxic, but once it is infiltrated to the pancreatic $\beta$-cells through the GLUT 2 transporter, alloxan is reduced to dialuric acid in the presence of different cellular reducing agents. The presence of both alloxan and its reduction product leads to the establishment of redox cycle for generation of superoxide radicals. The toxic action of alloxan on $\beta$ cells is initiated by free radicals formed by redox reactions. The intolerable rise in oxidative agents provokes necrosis of pancreatic $\beta$-cells known to be vulnerable to a redox imbalance. This suggests that alloxan diabetogenicity is a free radical-mediated process. Furthermore, alloxan toxicity is related to animal death due to fatal hypoglycemic convulsions. This study shows that oral administration of synthesised benzothiazole derivatives prevented the diabetogenic effect of alloxan; this is possibly due to the 
presence of antioxidant compounds, which act by inhibiting alloxaninduced free radicals production $[18,19]$.

All the synthesised derivatives were confirmed by FTIR, ${ }^{1} \mathrm{H}$ NMR, ${ }^{13} \mathrm{CNMR}$ and mass spectroscopy method. The animals showing blood glucose range of 200-400 $\mathrm{mg} \mathrm{dL}^{-1}$ were used for the experiment and the hyperglycemia was confirmed after $72 \mathrm{~h}$ of alloxan monohydrate administration (i. p.). It has been found that oral administration of synthesised compounds specifically $7 \mathrm{~d}, 7 \mathrm{~g}, 7 \mathrm{~h}$ and $7 \mathrm{l}$ at a defined dose of $350 \mathrm{mg} / \mathrm{kg}$ b.w. caused a more significant reduction in blood glucose than other compounds in diabetic rats. However, the compound $7 \mathrm{~d}$ exerted maximum glucose lowering effects whereas $7 \mathrm{i}$ showed minimum glucose lowering effects. This study reveals the result of test groups when significantly compared with positive control (alloxan $120 \mathrm{mg} / \mathrm{kg}$ ) p. o. and standard glibenclamide 10 $\mathrm{mg} / \mathrm{kg}$ (p. o.). In addition, it is necessary to ensure, to determine the exact mechanism by applying other in vitro methods for evaluation of the antidiabetic activity. Effective blood glucose control is the key for preventing or reversing diabetic complications and improving the quality of life in patients with diabetes. Thus, sustained reduction in hyperglycemia will decrease the risk of developing microvascular complications and most likely reduce the risk of macrovascular complications [20].

Table 3: Antidiabetic activity of synthesised compounds 7(a-l)

\begin{tabular}{|c|c|c|c|c|c|c|}
\hline \multirow[t]{2}{*}{ S. No. } & \multirow{2}{*}{$\begin{array}{l}\text { Treatment } \\
(350 \mathrm{mg} / \mathrm{kg} \text { b. w p. o) }\end{array}$} & \multicolumn{4}{|c|}{ Blood glucose level (mg/dl) } & \multirow[t]{2}{*}{$\%$ reduction in blood glucose } \\
\hline & & $0^{\text {th }}$ day & $7^{\text {th }}$ day & $14^{\text {th }}$ day & $21^{\text {st }}$ day & \\
\hline 1. & Normal control & $105.09 \pm 1.2$ & $102 \pm 0.3$ & $102 \pm 0.9$ & $100 \pm 1.2$ & - \\
\hline 2. & Diabetic positive control & $274 \pm 1.3$ & $273 \pm 1.6$ & $271 \pm 1.4$ & $270 \pm 1.9$ & - \\
\hline 3. & Glibenclamide $10 \mathrm{mg} / \mathrm{kg}$ & $278 \pm 2.1$ & $219 \pm 2.4$ & $168 \pm 1.8$ & $95 \pm 1.7$ & $65.82 \%$ \\
\hline 4. & $7 a$ & $274 \pm 1.4$ & $243 \pm 2.8$ & $206 \pm 1.3$ & $158 \pm 2.3$ & $42.33 \%$ \\
\hline 5. & $7 \mathrm{~b}$ & $271 \pm 2.7$ & $235 \pm 3.1$ & $191 \pm 0.8$ & $144 \pm 3.3$ & $46.86 \%$ \\
\hline 6. & $7 \mathrm{c}$ & $270 \pm 1.9$ & $246 \pm 2.7$ & $199 \pm 1.9$ & $161 \pm 1.5$ & $40.37 \%$ \\
\hline 7. & $7 d$ & $279 \pm 3.6$ & $222 \pm 3.4^{*}$ & $167 \pm 2.2^{*}$ & $102 \pm 1.8 \cdots$ & $63.44 \%$ \\
\hline 8. & $7 e$ & $281 \pm 2.8$ & $238 \pm 2.9$ & $192 \pm 1.3$ & $149 \pm 4.1$ & $46.97 \%$ \\
\hline 9. & $7 f$ & $272 \pm 1.7$ & $254 \pm 3.2$ & $205 \pm 1.6$ & $159 \pm 2.9$ & $41.54 \%$ \\
\hline 10. & $7 g$ & $283 \pm 1.3$ & $230 \pm 2.5$ & $174 \pm 1.4$ & $110 \pm 3.1 \cdots$ & $61.13 \%$ \\
\hline 11. & $7 \mathrm{~h}$ & $281 \pm 4.2$ & $235 \pm 2.7^{\circ}$ & $179 \pm 1.5$ & $114 \pm 2.6^{\circ}$ & $59.43 \%$ \\
\hline 12. & $7 \mathrm{i}$ & $276 \pm 3.4$ & $250 \pm 2.2$ & $210 \pm 0.5$ & $165 \pm 3.2$ & $40.21 \%$ \\
\hline 13. & $7 j$ & $273 \pm 1.6$ & $240 \pm 2.6$ & $192 \pm 2.2$ & $136 \pm 2.4$ & $50.18 \%$ \\
\hline 14. & $7 \mathrm{k}$ & $269 \pm 2.8$ & $244 \pm 2.3$ & $199 \pm 1.9$ & $146 \pm 2.1$ & $45.72 \%$ \\
\hline 15. & 71 & $274 \pm 1.5$ & $242 \pm 2.5^{\prime \prime}$ & $186 \pm 1.1$ & $123 \pm 1.8^{*}$ & $55.10 \%$ \\
\hline
\end{tabular}

$\mathrm{n}=6$, Values are expressed as mean \pm SD, $\mathrm{P}<0.05,{ }^{\circ} \mathrm{P}<0.01,{ }^{\cdots} \mathrm{P}<0.001$-significant compared to standard group

Researchers also synthesised a novel series of substituted (E)-3(Benzo[d]thiazol-2-ylamino) phenylprop-2-en-1-ones and evaluated for their antidiabetic activity [21]. Selective inhibitors of 11betahydroxysteroid dehydrogenase type 1 (11ß-HSD1) have considerable potential as treatments for metabolic diseases, such as diabetes mellitus type 2 or obesity. Moreover, a series of novel benzothiazole derivatives were synthesised and studied for their inhibitory activities against 11-HSD1 from human hepatic microsomes measured using a radioimmunoassay (RIA) method [22]. Benzothiazole derivatives of thiazolidinones were synthesised and assayed for activity on Peroxisome proliferator-activated receptor (PPAR) subtypes and inhibitory activity of NO production in lipopolysaccharide-activated macrophages. Most of the compounds were identified as PPAR $\gamma$ agonist, indicating their potential as drug candidate for diabetes [23].

\section{CONCLUSION}

The diabetic hyperglycemia induced by alloxan produces an elevation of plasma levels of glucose, which is considered significant marker of renal dysfunction. The benzothiazole derivatives were synthesised by sequencing scheme and subsequently confirmed by different spectroscopy methods. The different physicochemical properties were characterised and then the synthesised derivatives were evaluated for their anti-diabetic activity in an alloxan-induced diabetic rat model. Amongst all these synthesised derivatives compound $7 \mathrm{~d}$ demonstrated more potent anti-diabetic activity at $350 \mathrm{mg} / \mathrm{kg} \mathrm{p}$. o. and would be of better use in drug development to combat the metabolic disorder in future.

\section{ACKNOWLEDGEMENT}

We are thankful to Punjab University Chandigarh and CDRI Lucknow, India for sophisticated analytical instrument facility. We are also thankful to Head, Sapience Bioanalytical Laboratory Bhopal, India for performing the antidiabetic activity. The authors have declared no conflict of interest.

\section{ABBREVIATION}

Stretch: Stretching, Sym: Symmetric, Asym: Asymmetric.

\section{CONFLICTS OF INTERESTS}

All the authors have no conflicts of interests.

\section{REFERENCES}

1. Jain R, Jain P, Jain P. A review on treatment and prevention of diabetes mellitus. Int J Curr Pharm Res 2016;8:16-8.

2. Malang M, Kayal G, Kharia A. Comparative study of heterocyclics in the treatment of diabetes. World J Pharm Pharm Sci 2015;4:796-811.

3. Rupeshkumar M, Kavitha K, Haldar PK. Role of herbal plants in the diabetes mellitus therapy: an overview. Int J Appl Pharm 2014;6:1-3.

4. Yadav PS, Prakash D, Senthilkumar GP. Benzothiazole: different methods of synthesis and diverse biological activities. Int J Pharm Sci Drug Res 2011;3:1-7.

5. Mariappan G, Prabhat P, Sutharson L, Banerjee J, Patangia U, Nath S. Synthesis and antidiabetic evaluation of benzothiazole derivatives. J Korean Chem Soc 2012;56:251-6.

6. Suter $H$, Zutter $H$. Studies concerning benzothiazoles as eventual oral antidiabetics. Helv Chim Acta 1967;50:1084.

7. Diaz HM, Molina RV, Andrade RO, Coutino DD, Medina-Franco JL, Webster SP, et al. Antidiabetic activity of N-(6-substituted1,3-benzothiazol-2-yl) benzenesulfonamides. Bioorg Med Chem Lett 2008;18:2871-7.

8. Shrivastava SD, Sen JP. Synthesis and biological evaluation of 2aminobenzothiazole derivatives. Indian J Chem 2008;47B:1583-6.

9. Gupta A, Rawat S. Synthesis and cyclisation of benzothiazole: a review. J Curr Pharm Res 2010;3:13-23.

10. Chaudhary M, Pareek D, Pareek PK, Kant R, Ojha KG, Pareek A. Synthesis of some new biologically active benzothiazole derivatives containing benzimidazole and imidazoline moieties. Bull Korean Chem Soc 2011;32:131-6.

11. Kapratwar SB. Novel synthesis and ring closure reactions of 3hydrazino-1, 2, 4 triazolo[3,4-b]benzothiazole. Indian J Chem 2005;44B:625-7. 
12. El-Shahawy AS, Kandeel MM, Youssef MSKZ. Electronic spectroscopic studies of some 3-methyl-5-pyrazolone derivatives. Phys Chem Leipzig 1990;271:1047.

13. Pareek D, Chaudhary M, Pareek PK, Ojha KG, Pareek A. Synthesis and biological evaluation of substituted 3(benzothiazolyl)-1, 3-thiazolidine-4-ones. Int J Pharm Pharm Sci 2014;6:438-55.

14. OECD. Guideline on acute oral toxicity (AOT) environmental health and safety monograph series on testing and adjustment; 2008. p. 425.

15. Kesari AN, Gupta RK, Singh SK, Diwakar S, Watal G. Hypoglycemic and antihyperglycemic activity of Aegal marmelos seed extracts in normal and diabetic rats. J Ethnopharmacol 2006;107:374-9.

16. Kumar AY, Kumar N, Handral M, Talwar S, Dhayabaran D. Hypoglycaemic and anti-diabetic activity of stem bark extracts Erythrina indica in normal and alloxan-induced diabetic rats. Saudi Pharm J 2011;19:35-42.

17. Abhinayani G, Kishore N, Benazir F, Agarwal P. Anti-diabetic activity of Epipremnum aureum. $l$ in normal and alloxaninduced diabetic rats. Asian J Pharm Clin Res 2016;9:89-92.

18. Muhtadia, Primariantia AU, Sujonoa TA. Antidiabetic activity of durian (Durio zibethinus Murr.) and rambutan (Nephelium lappaceum L.) fruit peels in alloxan diabetic rats. Procedia Food Sci 2015;3:255-61.

19. Berraaouan A, Abderrahim Z, Hassane M, Abdelkhaleq L, Mohammed A, Mohamed B. Evaluation of the protective effect of cactus pear seed oil (Opuntia ficus-indica L. MILL.) against alloxan induced diabetes in mice. Asian Pacific J Trop Med 2015;8:532-7.

20. Jarald EE, Joshi SB, Jain DC. Antidiabetic activity of flower buds of Michelia champaca Linn. Indian J Pharmacol 2008;40:256-60.

21. Patil VS, Nandre KP, Ghosh S. Synthesis, crystal structure and antidiabetic activity of substituted (E)-3-(Benzo [d]thiazol-2ylamino) phenylprop-2-en-1-one. Eur J Med Chem 2013;59:304-9.

22. Su X, Vicker N, Ganeshapillai D, Smith A, Purohit A, Reed MJ, et al. Benzothiazole derivatives as novel inhibitors of human $11 \beta$ hydroxysteroid dehydrogenase type 1. Mol Cell Endocrinol 2006;248:214-7.

23. Jeon R, Kim Y, Cheon Y, Ryu J. Synthesis and biological activity of [[(hetero cyclo amino)alkoxy]benzyl]-2, 4-thiazolidinediones as PPAR $\gamma$ agonists. Arch Pharmacal Res 2006;29:394-9.

\section{How to cite this article}

- Sunil Kumar, DS Rathore, Gopal Garg, Kapil Khatri, Rahul Saxena, Sanjeev K Sahu. Synthesis and evaluation of some benzothiazole derivatives as antidiabetic agents. Int J Pharm Pharm Sci 2017;9(2):60-68. 